\title{
Drawing Graphs in the Hyperbolic Plane
}

\author{
Bojan Mohar* \\ Department of Mathematics, University of Ljubljana \\ 1111 Ljubljana, Slovenia \\ bojan.mohar@uni-lj.si
}

\begin{abstract}
It is shown how one can draw graphs on surfaces of negative Euler characteristic by using hyperbolic geometry and hyperbolic circle packing representations. The same approach applies to drawings of hyperbolic tessellations.
\end{abstract}

\section{Introduction}

The purpose of this note is to offer a new approach for drawing graphs embedded in a surface $\Sigma$ with negative Euler characteristic. Each such embedding can be changed by a homeomorphism to an embedding in a Riemann surface $\Sigma_{0}$ homeomorphic to $\Sigma$ of constant negative curvature $\kappa<0$ (say $\kappa=-1$ ) such that all edges of the graph become "straight line segments" (i.e., geodesic segments) on $\Sigma_{0}$. To get such a representation, we suggest using the primal-dual circle packing algorithm (abbreviated PDCP) which is presented in [9] (originally introduced by Lovász, see [3]) for the Euclidean plane and in [8] (see also [4], [5]) for the hyperbolic case treated in this paper. The PDCP algorithm determines a convex embedding in $\Sigma_{0}$ if (the universal cover of) the given graph $G$ embedded in $\Sigma$ is 3-connected. By considering the universal cover $\widetilde{\Sigma}_{0}$ of $\Sigma_{0}$, the problem of drawing $G$ on $\Sigma_{0}$ transforms to the problem of drawing graphs in the hyperbolic plane $\mathbb{H}^{2} \approx \widetilde{\Sigma}_{0}$ and then identifying the fundamental domain of $\Sigma_{0}$ in $\mathbb{H}^{2}$.

In Sections 2 and 3 we present two basic models of the hyperbolic plane - the upper half-plane and the unit disk model. Further on, we present the relation between the Euclidean and the hyperbolic geometry of the upper half-plane which enables us to develop the basic graph drawing primitives for drawings in $\mathbb{H}^{2}$. At the end we add a few examples.

It is to be hoped that our approach will stimulate further research related to drawings of graphs in the hyperbolic geometry. Our main motivation for this type of graph drawings are representations of graphs embedded in higher genus surfaces and drawings of hyperbolic tessellations. A related application to draw and navigate Internet sites and their connections using hyperbolic geometry was explored by Munzner [11].

For further reading on the hyperbolic geometry we refer the reader to [6], [12], [13], [14], [15].

\footnotetext{
* Supported in part by the Ministry of Science and Technology of Slovenia, Research Project J1-0502-0101-98.
} 


\section{The Poincaré Half-Plane}

The Euclidean plane $\mathbb{R}^{2}$ is endowed with the Euclidean metric

$$
d s^{2}=d x^{2}+d y^{2} .
$$

It is convenient to identify $\mathbb{R}^{2}$ with $\mathbb{C}$, the set of all complex numbers, so that the point $(x, y) \in \mathbb{R}^{2}$ is identified with $z=x+i y \in \mathbb{C}$.

The Poincaré half-plane $\mathbb{H}^{2}=\left\{(x, y) \in \mathbb{R}^{2} \mid y>0\right\}$ is the upper half-plane endowed with the hyperbolic metric

$$
d s^{2}=\frac{d x^{2}+d y^{2}}{y^{2}} .
$$

If $\gamma(t)=x(t)+i y(t), a \leq t \leq b$, is a $C^{1}$-curve in $\mathbb{H}^{2}$, then its hyperbolic length $\ell(\gamma)$ is defined as

$$
\ell(\gamma)=\int_{\gamma} \frac{\sqrt{d x^{2}+d y^{2}}}{y} .
$$

The geodesic lines of $\mathbb{H}^{2}$ (also called hyperbolic straight lines) are either

(a) Euclidean semicircles with the center on the $x$-axis, or

(b) Euclidean straight lines in $\mathbb{H}^{2}$ which are perpendicular to the $x$-axis.

See Figure 1.

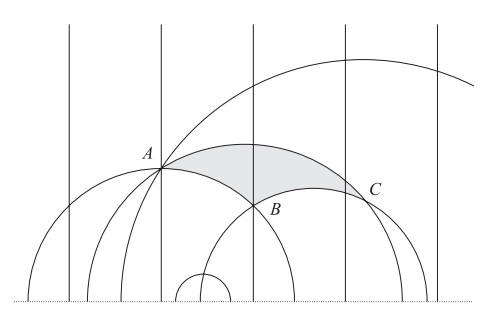

Fig. 1. Geodesic lines in $\mathbb{H}^{2}$

For any two points $z, w \in \mathbb{H}^{2}$, there is a unique hyperbolic straight line $P$ containing them. The hyperbolic distance of $z$ and $w$, denoted by dist $\mathbb{H}(z, w)$, is the hyperbolic length of the segment of $P$ from $z$ to $w$.

If $A, B, C$ are points in $\mathbb{H}^{2}$, then the hyperbolic line segments joining $A, B$, and $C$ determine the hyperbolic triangle $\triangle(A B C)$. In Figure 1, one of such triangles is displayed.

Theorem 1. The rigid motions of $\mathbb{H}^{2}$ are precisely the transformations of the following forms:
(a) $f(z)=\frac{a z+b}{c z+d}$
or
(b) $f(z)=\frac{-a \bar{z}+b}{-c \bar{z}+d}$

where $a, b, c, d$ are real numbers and $a d-b c>0$. 
Let us mention two special cases. The hyperbolic rotation of $\mathbb{H}^{2}$ by the angle $2 \vartheta$ about the point $i=(0,1)$ is the mapping

$$
R_{\vartheta}(z)=\frac{\cos \vartheta z+\sin \vartheta}{-\sin \vartheta z+\cos \vartheta}
$$

The translation which maps $(a, b) \in \mathbb{H}^{2}$ to the point $(c, d) \in \mathbb{H}^{2}$ is the map

$$
T_{a, b, c, d}(z)=\frac{d z+(b c-a d)}{b} .
$$

Two geometric figures $A, B$ in $\mathbb{H}^{2}$ (subsets of $\mathbb{H}^{2}$ ) are congruent if there is a rigid motion of $\mathbb{H}^{2}$ which transforms $A$ into $B$.

Let $z=(x, y) \in \mathbb{H}^{2}$ and $r \in \mathbb{R}^{+}$. The (hyperbolic) circle of radius $r$ centered at $z$ is the set

$$
C(z, r)=\left\{w \in \mathbb{H}^{2} \mid \operatorname{dist}_{\mathbb{H}}(z, w) \leq r\right\} .
$$

The Euclidean circle of radius $r$ centered at $z$ is defined as the set

$$
C_{E}(z, r)=\{w \in \mathbb{C}:|z-w| \leq r\} .
$$

Theorem 2. Every hyperbolic circle is also a Euclidean circle. If $z=(x, y) \in$ $\mathbb{H}^{2}$ and $r \in \mathbb{R}^{+}$, then $C(z, r)=C_{E}\left(z^{\prime}, r^{\prime}\right)$ where $z^{\prime}=x+i y^{\prime}, y^{\prime}=y \operatorname{ch}(r)$, $r^{\prime}=y \operatorname{sh}(r)$.

Proof. We shall give only the proof of the second statement. Let $z_{1}=\left(x, y_{1}\right)$ and $z_{2}=\left(x, y_{2}\right)$ be the points in $\mathbb{H}^{2}$ at hyperbolic distance $r$ from $z$ which have the same first coordinate $x$ as $z$ and where $y_{1}<y<y_{2}$. By (1) we easily get $y_{1}=y \exp (-r)$ and $y_{2}=y \exp (r)$. This implies that the Euclidean circle $C_{E}\left(z^{\prime}, r^{\prime}\right)$ has radius $r^{\prime}=\left(y_{2}-y_{1}\right) / 2=y \operatorname{sh}(r)$ and center $z^{\prime}=\left(x,\left(y_{1}+y_{2}\right) / 2\right)$, so that $y^{\prime}=y \operatorname{ch}(r)$.

If $A, B, C$ are points in $\mathbb{H}^{2}$, then the hyperbolic line segments joining $A, B$, and $C$ determine the hyperbolic triangle $\Delta(A B C)$. In Figure 1 one of such triangles is displayed. It is well known that the sum of the angles $\alpha, \beta, \gamma$ of a hyperbolic triangle is always less that $\pi$. It is also known that for any positive real numbers $\alpha, \beta, \gamma$ whose sum is $<\pi$ there is a hyperbolic triangle with angles $\alpha, \beta, \gamma$. Moreover, any two such triangles are congruent and have the same hyperbolic area (which is equal to $\pi-\alpha-\beta-\gamma$ ).

Suppose that $\Delta$ is a hyperbolic triangle with angles $\alpha, \beta, \gamma$ and lengths of its sides equal to $a, b, c$ (where the side of length $a$ is opposite the angle $\alpha$, etc.). These quantities are then related by the Hyperbolic Law of Cosines

$$
\cos \alpha=\frac{\operatorname{ch} b \cdot \operatorname{ch} c-\operatorname{ch} a}{\operatorname{sh} b \cdot \operatorname{sh} c} \quad \text { and } \quad \operatorname{ch} a=\frac{\cos \beta \cdot \cos \gamma+\cos \alpha}{\sin \beta \cdot \sin \gamma}
$$

and by the Hyperbolic Law of Sines

$$
\frac{\sin \alpha}{\operatorname{sh} a}=\frac{\sin \beta}{\operatorname{sh} b}=\frac{\sin \gamma}{\operatorname{sh} c}
$$

In the special case where $\gamma=\frac{\pi}{2}$ (the right triangle), we get

$$
\operatorname{ch} c=\operatorname{ch} a \cdot \operatorname{ch} b=\operatorname{ctg} \alpha \cdot \operatorname{ctg} \beta .
$$




\section{The Unit Disk Model of the Hyperbolic Plane}

The Möbius transformation

$$
U(z)=\frac{i z+1}{z+i}
$$

maps $\mathbb{H}^{2}$ bijectively onto the (open) unit disk $\mathbb{D}^{2}$ in $\mathbb{C}$. The inverse mapping is

$$
U^{-1}(w)=\frac{i w-1}{-w+i}
$$

The geodesics of $\mathbb{H}^{2}$ are mapped by $U(z)$ onto circular arcs with their endpoints on the boundary of $\mathbb{D}^{2}$ which meet the boundary of $\mathbb{D}^{2}$ perpendicularly, see Figure 2 below.

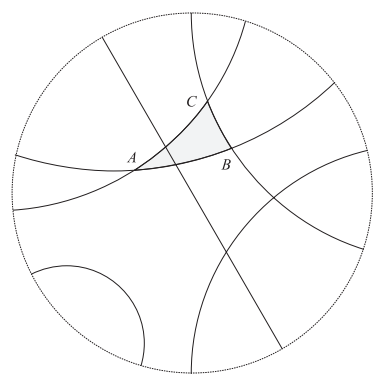

Fig. 2. Geodesic lines in the hyperbolic unit disk model

The mapping $U(z)$ determines the hyperbolic geometry on $\mathbb{D}^{2}$ which has the following Riemann metric

$$
d s^{2}=\frac{4\left(d x^{2}+d y^{2}\right)}{\left(1-x^{2}-y^{2}\right)^{2}} .
$$

If $\gamma(t)=x(t)+i y(t), a \leq t \leq b$, is a $C^{1}$-curve in $\mathbb{D}^{2}$, then its hyperbolic length $\ell(\gamma)$ is defined as

$$
\ell(\gamma)=\int_{\gamma} \frac{2 \sqrt{d x^{2}+d y^{2}}}{1-x^{2}-y^{2}}
$$

Theorem 3 below determines the rigid motions of $\mathbb{D}^{2}$ :

Theorem 3. The rigid motions of $\mathbb{D}^{2}$ are precisely the transformations of the following forms:
(a) $g(z)=\frac{a z+\bar{c}}{c z+\bar{a}} \quad$ or
(b) $g(z)=\frac{-a \bar{z}+\bar{c}}{-c \bar{z}+\bar{a}}$

where $a$ and $c$ are any complex numbers such that $|a|>|c|$. 
Observe that $U(z)$ is a Möbius transformation. Since all Möbius transformations map circles (and halfspaces) to circles (and halfspaces), Theorem 2 implies:

Theorem 4. Every hyperbolic circle in $\mathbb{D}^{2}$ of radius $r$ and with center $w \in$ $\mathbb{D}^{2}$ corresponding to the hyperbolic disk metric (11) is also a Euclidean circle $C_{E}\left(w^{\prime}, r^{\prime}\right)$, where $w^{\prime}=\frac{-4 e^{r} y}{-\left(1+e^{r}\right)^{2}+\left(-1+e^{r}\right)^{2} y^{2}} \frac{w}{|w|}$ and $r^{\prime}=\frac{\left(-1+e^{2 r}\right)\left(-1+y^{2}\right)}{-\left(1+e^{r}\right)^{2}+\left(-1+e^{r}\right)^{2} y^{2}}$.

\section{Hyperbolic Primal Dual Circle Packings}

Let $\Sigma$ be a surface. A map on $\Sigma$ is a pair $(G, \Sigma)$ where $G$ is a connected graph that is 2-cell embedded in $\Sigma$. Given a map $M=(G, \Sigma)$, a circle packing of $M$ is a set of (geodesic) circles in a Riemannian surface $\Sigma_{0}$ of constant curvature that is homeomorphic to $\Sigma$, one circle for each vertex of $G$, such that the following conditions are fulfilled:

(i) the interiors of circles are pairwise disjoint open disks,

(ii) for each edge $u v \in E(G)$, the circles corresponding to $u$ and $v$ touch, and

(iii) by putting a vertex $v_{D}$ in the centre of each circle $D$ and joining $v_{D}$ by geodesics with all points on the boundary of $D$ where the other circles touch $D$ (or where $D$ touches itself), we get a map on $\Sigma_{0}$ which is isomorphic to $M$.

Because of (iii) we also say to have a circle packing representation of $M$. The obtained map on $\Sigma_{0}$ is said to be a straight-line representation of $M$. Simultaneous circle packing representations of a map $M$ and its dual map $M^{*}$ are called a primal-dual circle packing representation of $M$ if for any two edges

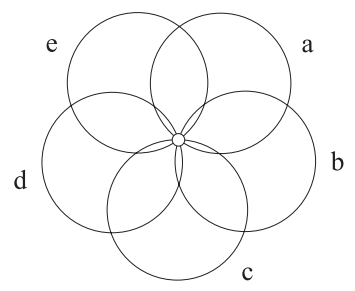

Fig. 3. The local rotation of $G_{0}$

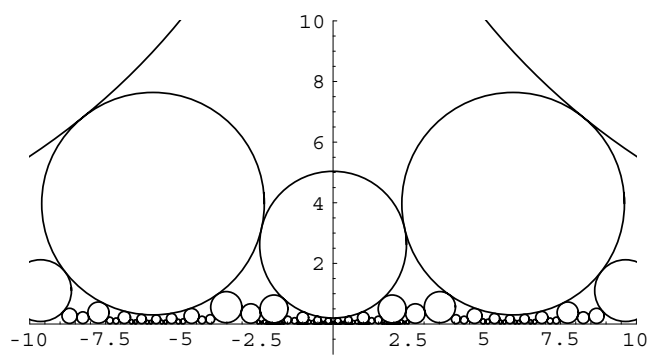

Fig. 4. The circle packing of $G_{0}$ in $\mathbb{H}^{2}$ 
$e=u v \in E(M)$ and $e^{*}=u^{*} v^{*} \in E\left(M^{*}\right)$ which are dual to each other, the circles $C_{u}, C_{v}$ corresponding to $e$ touch at the same point as the $\operatorname{circles} C_{u^{*}}, C_{v^{*}}$ of $e^{*}$, and $C_{u}, C_{u^{*}}$ cross each other at that point perpendicularly. Having a primal-dual circle packing representation, each pair of dual edges intersects at the right angle. The obtained representations of the maps $M$ and $M^{*}$ on $\Sigma_{0}$ are easily seen to be convex, i.e., if $x, y$ are points in the same face $F$ of $M\left(\right.$ or $M^{*}$ ), then in $F$ there is a geodesic (not necessarily a shortest one) joining $x$ and $y$.

It was proved by Koebe [7], Andreev [1], [2], and Thurston [16] that if $M$ is a triangulation, then it admits a circle packing representation (some of these results apply only for the 2-sphere). The proofs of Andreev and Thurston are existential (using a fixed point theorem) but Colin de Verdière [4], [5] found a constructive proof by means of a convergent process. Mohar [8] found a polynomial time algorithm that for a given map $M$ (whose universal cover has 3-connected graph) and for a given $\varepsilon=10^{-t}$ finds an $\varepsilon$-approximation for a circle packing of $M$ into a surface of constant curvature (either $+1,0$, or -1 ). The time used by that algorithm is polynomial in the size of the input $(=|V(M)|+|E(M)|+t)$.

\section{$5 \quad$ Drawing Primitives}

To draw graphs in the hyperbolic plane as suggested in the introduction we have to implement drawing primitives for drawing (hyperbolic) circles and hyperbolic line segments. We assume that we have a primitive for drawing Euclidean circles and Euclidean circular arcs: $\mathbb{R}^{2}$.

$\operatorname{Circle}(a, b, r)$ : Draws the Euclidean circle with radius $r$ centered at $(a, b) \in$

CircularArc $(a, b, r, \alpha, \beta)$ : Draws the circular arc of Circle $(a, b, r)$ corresponding to the angles from $\alpha$ to $\beta$.

LineSegment $(a, b, c, d)$ : Draws the Euclidean straight line segment from $(\mathrm{a}, \mathrm{b})$ to $(\mathrm{c}, \mathrm{d})$.

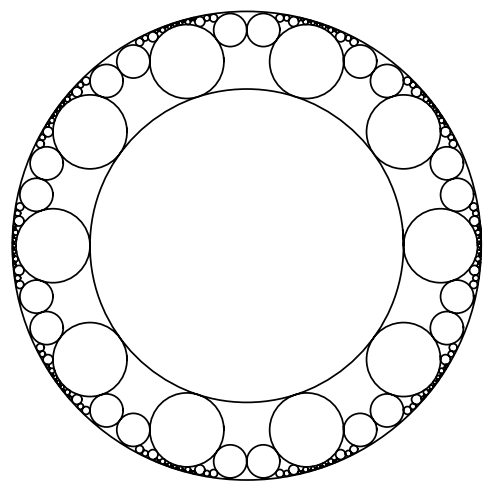

Fig. 5. The circle packing of $G_{0}$ in $\mathbb{D}^{2}$ 
We shall describe only the upper half-plane model. The unit disk model primitives are obtained by simply applying the Möbius transformation (9).

\subsection{Circles}

HyperbolicCircle $(\mathrm{x}, \mathrm{y}, \mathrm{r})$ : Draws the hyperbolic circle with radius $\mathrm{r}$ centered at $(\mathrm{x}, \mathrm{y}) \in \mathbb{H}^{2}$. This primitive can be expressed as $\operatorname{Circle}\left(\mathrm{x}, \mathrm{y}^{\prime}, \mathrm{r}^{\prime}\right)$ where $\mathrm{y}^{\prime}$ and $r^{\prime}$ are given by Theorem 2 .

\subsection{Hyperbolic Line Segments}

HyperbolicLine ( $a, b, c, d)$ : Draws the hyperbolic line segment from $(a, b) \in \mathbb{H}^{2}$ to $(c, d) \in \mathbb{H}^{2}$. If $\mathrm{a}=\mathrm{c}$, this is just a vertical line segment. Otherwise, this is the circular arc of the Euclidean circle centered at $(x, 0)$ where $x=\left(c^{2}+d^{2}-a^{2}-\right.$ $\left.b^{2}\right) /(2(c-a))$.

HyperbolicLineTangent $(\mathrm{a}, \mathrm{b}, \alpha, 1)$ : Draws the hyperbolic line segment from $(\mathrm{a}, \mathrm{b}) \in \mathbb{H}^{2}$ of length 1 which starts at $(\mathrm{a}, \mathrm{b})$ under the angle $\alpha$ with respect to the $x$-axis. This operation is easiest to implement by using hyperbolic rigid motions to determine the endpoint $(c, d)$ of the segment (compose the translation $T_{a, b, 0,1}$ defined by (3), the rotation $R_{\varphi}$ by the angle $2 \varphi, \varphi=\frac{1}{2}\left(\frac{\pi}{2}-\alpha\right)$, about the point $i=(0,1) \in \mathbb{H}^{2}$, determine the modified end of the line segment - the point on the $y$-axis above $i$ at hyperbolic distance 1 , and map back). Finally, we just draw HyperbolicLine (a,b,c,d). The same operation can be done directly by using CircularArc $\left(\mathrm{x}, 0, \mathrm{r}, \varphi_{1}, \varphi_{2}\right)$ where $x=a+b / \operatorname{tg} \alpha$, $r=\sqrt{(x-a)^{2}+b^{2}}, \varphi_{1}=\frac{\pi}{2}+\alpha$, and $\varphi_{2}=2 \operatorname{arctg}\left(\operatorname{tg}\left(\frac{\pi}{4}+\frac{\alpha}{2}\right) / 1\right)$. (Other formulae apply if $\alpha \in\left\{\frac{\pi}{2}, \frac{3 \pi}{2}\right\}$.)

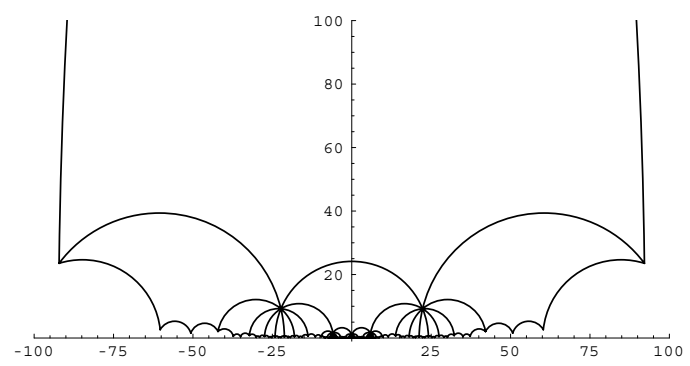

Fig. 6. The straight line drawing of $G_{0}$ in $\mathbb{H}^{2}$

\section{Drawing in the Hyperbolic Plane}

For the sake of simplicity we shall assume that $G_{0}$ is a graph embedded in some surface of negative Euler characteristic such that the graph of its universal cover 
is 3-connected. (Otherwise we extend $G_{0}$ to a triangulation whose graph (and the graph of the universal cover) is necessarily 3-connected.)

Drawing algorithm consists of the following steps:

(1) Input the graph $G_{0}$ and the combinatorial description of its embedding $\Pi_{0}$ (see, e.g., [10]).

(2) Determine the $\Pi_{0}$-facial walks and construct the vertex-face incidence graph $G$ (cf. [8]).

(3) Determine the radii $r_{v}(v \in V(G))$ of the primal-dual circle packing (cf. [8]).

(4) Start the BFS of the universal cover $\tilde{G}$ of $G$ and draw its faces (respectively, the edges of $G_{0}$ or the circles of the circle packing of $G_{0}$ ) one after another until all the faces (respectively, edges or circles) have been drawn (at least) once. Identifying the first drawing of each of the faces gives the fundamental polygon of the surface.

(5) For a representation in the unit disk model, transform the drawing by applying the mapping $U(z)$ given by equation (9).

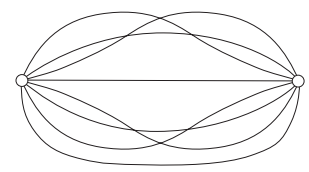

Fig. 7. The local rotation of $G_{1}$

\section{$7 \quad$ Examples}

Let $G_{0}$ be the graph with a single vertex $v$ and with five loops a,b,c,d,e. Consider its embedding determined by the local rotation shown in Figure 3. This embedding has two facial walks, $A=$ adbec and $B=$ acebd. By Euler's formula, this is an embedding in the double torus. The vertex-face incidence graph has three vertices $v, A, B, 5$ edges joining $v$ and $A$, and 5 edges joining $v$ and $B$. The radii of the corresponding $\mathrm{PDCP}$ are $r_{v}=1.61692, r_{A}=r_{B}=1.06128$. Circle packings of $G_{0}$ in the hyperbolic upper half-plane and in the hyperbolic disk are shown in Figures 4 and 5, respectively. The corresponding straight line drawing in the hyperbolic plane is presented in Figure 6.

Figures 8 and 9 show the circle packing representations of the map $G_{1}$ shown in Figure 7.

\section{Acknowledgment}

The author is grateful to Arjana Žitnik for calculating the circle packing radii and for producing the actual circle packing drawings shown in Section 7. 


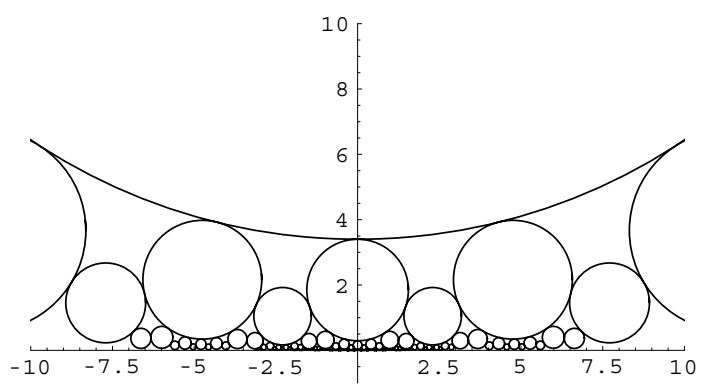

Fig. 8. The circle packing of $G_{1}$ in $\mathbb{H}^{2}$

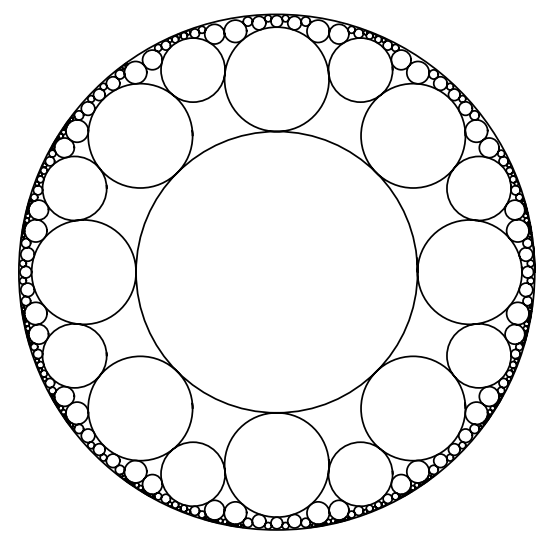

Fig. 9. The circle packing of $G_{1}$ in $\mathbb{D}^{2}$

\section{References}

1. E. M. Andreev, On convex polyhedra in Lobačevskii spaces, Mat. Sb. (N. S.) 81 (1970) 445-478; Engl. transl. in Math. USSR Sb. 10 (1970) 413-440.

2. E. M. Andreev, On convex polyhedra of finite volume in Lobačevskii space, Mat. Sb. (N. S.) 83 (1970) 256-260; Engl. transl. in Math. USSR Sb. 12 (1970) 255-259.

3. G. R. Brightwell, E. R. Scheinerman, Representations of planar graphs, SIAM J. Disc. Math. 6 (1993) 214-229.

4. Y. Colin de Verdière, Empilements de cercles: Convergence d'une méthode de point fixe, Forum Math. 1 (1989) 395-402.

5. Y. Colin de Verdière, Un principe variationnel pour les empilements des cercles, Invent. Math. 104 (1991) 655-669.

6. B. Iversen, Hyperbolic geometry, Cambridge Univ. Press, 1992.

7. P. Koebe, Kontaktprobleme auf der konformen Abbildung, Ber. Verh. Saechs. Akad. Wiss. Leipzig, Math.-Phys. Kl. 88 (1936) 141-164.

8. B. Mohar, Circle packings of maps in polynomial time, Europ. J. Combin. 18 (1997) 785-805. 
9. B. Mohar, Circle packings of maps - The Euclidean case, Rend. Sem. Mat. Fis. (Milano), in press.

10. B. Mohar, C. Thomassen, Graphs on Surfaces, Johns Hopkins Univ. Press, to appear.

11. T. Munzner, Drawing large graphs with H3Viewer and Site Manager (system demonstration), in "Graph Drawing '98", Lecture Notes in Computer Science, Springer-Verlag, 1998, pp. 384-393.

12. A. Ramsay, R. D. Richtmeyer, Introduction to Hyperbolic Geometry, SpringerVerlag, New York, 1995.

13. J. G. Ratcliffe, Foundations of Hyperbolic Manifolds, Springer-Verlag, New York, 1994.

14. D. M. Y. Sommerville, The Elements of Non-Euclidean Geometry, Dover, New York, 1958.

15. S. Stahl, The Poincaré Half-Plane, Jones and Barlett Publishers, Boston, London, 1993.

16. W. P. Thurston, The geometry and topology of 3-manifolds, Princeton Univ. Lect. Notes, Princeton, NJ. 